\title{
Retinal nerve fiber layer defects in the presence of a physiological cup to disc ratio - a case series
}

\author{
Carlos Eduardo Rivera $\mathbb{1}^{1-4}$, Juan Carlos Aristizabal $\mathbb{D}^{1,2}$, Edgar Muñoz $\mathbb{1}^{1,5}$, Ankur Seth $\mathbb{1}^{1,3,6}$ \\ ${ }^{1}$ Collective Innovations Colombia, Cali, Colombia \\ ${ }^{2}$ Pontificia Universidad Javeriana, Cali, Colombia \\ ${ }^{3}$ GSR Medical Center, Cali, Colombia \\ ${ }^{4}$ GSR International, United States \\ ${ }^{5}$ UT Health San Antonio, San Antonio, United States \\ ${ }^{6}$ University of Tennessee Health Science Center, United States
}

\begin{abstract}
Retinal nerve fiber layer (RNFL) defects are one of the earliest signs of glaucoma. Typically, these RNFL defects are associated with an increased cup/disc ratio and a thinning of the neuroretinal rim. When the cup/disc ratio is within normal limits, the observer can misdiagnose subtle RNFL defects and lead to an essential delay in diagnosis, which has negative visual consequences in these patients. We present a case series report addressing RNFL defects with a physiological cup/disc ratio.
\end{abstract}

KEY WORDS: glaucoma; retinal nerve fiber defects; cup/disc ratio

Ophthalmol J 2021; Vol. 6, 258-264

\section{INTRODUCTION}

Glaucoma is considered an optic neuropathy with a thinning of the peripapillary retinal nerve fiber layer and optic disc cupping secondary to retinal ganglion cell loss. One of the earliest signs of glaucoma are retinal nerve fiber layer (RNFL) defects [1]. A physiological or normal cup to disc ratio (C/D) is considered any $\mathrm{C} / \mathrm{D}$ lower than 0.7 , and it has a significant variability among the population $[1,3]$.

Generally, structural changes in the neuroretinal rim and RNFL precede visual field (VF) defects in the early stages of glaucoma. Therefore, detecting structural changes in the neuroretinal rim and RNFL is important for early glaucoma detection
[2]. RNFL thinning is highly specific to glaucomatous optic neuropathy $[3,4]$.

We present a case series report addressing RNFL defects with a physiological cup/disc ratio.

\section{CLINICAL CASES}

This case series report was selected from an ongoing glaucoma prevalence study of 4,838 patients in Cali, Colombia. We selected patients with RNFL defects by red-free photographs in the presence of a regular cup/disc ratio. It is noteworthy that only 7 of 4838 patients met this criterion, emphasizing that this type of presentation is unusual. The mor-

CORRESPONDING AUTHOR:

Carlos Rivera, Holguines Trade Center, Cra 10 11-60, office 236, tel: (2) 3165225961, (572) 5546567; e-mail: carlosriverahoyos@gmail.com, crivera@grupogsr.co 


\section{A}

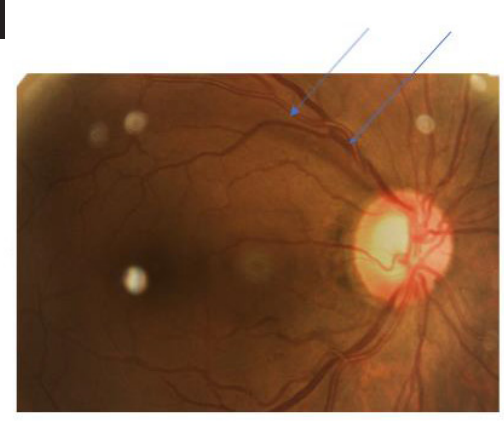

D



B

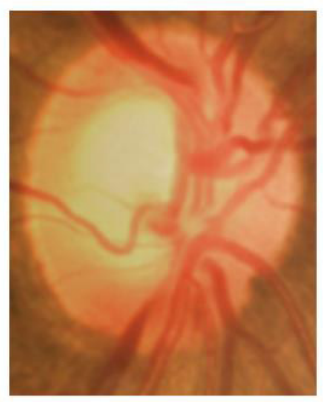

C

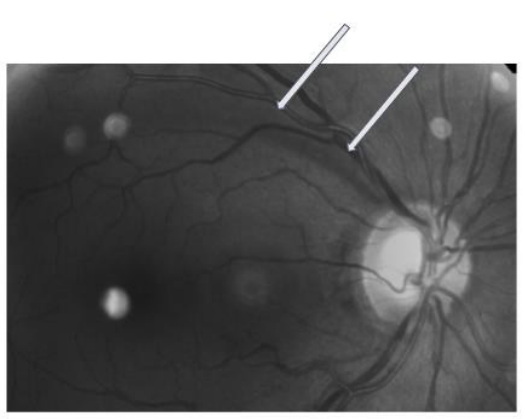

E

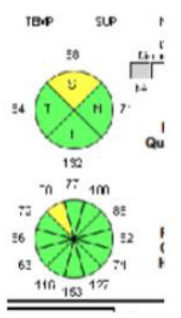

F

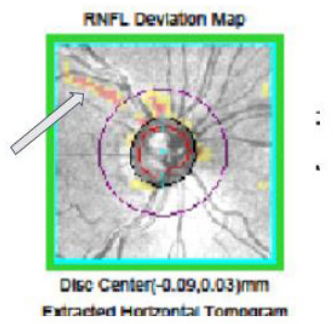

FIGURE 1. A. Right eye (RE) color fundus optic nerve image showing superior retinal nerve fiber layer (RNFL) defect (blue arrow);

B. First plane optic nerve showing physiologic cup/disc ratio; C. RE red-free optic nerve image showing superior RNFL defect (white arrow); D. RNFL thickness map showing superior RNFL defect (white arrow); E. RNFL clock hour meridians showing superior pole thinning. RNFL deviation map showing in red RNFL defect (white arrow)

phometric parameters of the optic disc and RNFL thickness were measured using ZEISS CIRRUS ${ }^{\mathrm{rm}}$ HD-OCT Model 4000 optical coherence tomography (Carl Zeiss Inc., Dublin, CA, USA).

\section{Case 1}

56-year-old female patient with an 8-year diagnosis of open-angle glaucoma in treatment with latanoprost: VA was 20/25 in both eyes; both eyes presented a normal anterior segment evaluation, IOP was $16 \mathrm{~mm} \mathrm{Hg}$ and $15 \mathrm{~mm} \mathrm{Hg}$, respectively. Fundus examination showed a cup/disc (C/D) ratio of 0.5 in the right eye $(\mathrm{RE})$ and $\mathrm{C} / \mathrm{D}$ ratio of 0.5 in the left eye (LE) (Fig. 1).

\section{Case 2}

58-year-old male patient with a 7-year diagnosis of open-angle glaucoma in treatment with timolol/dorzolamide combination: VA was $20 / 25$ in both eyes; anterior segment examination was normal in both eyes, IOP was $12 \mathrm{~mm} \mathrm{Hg}$ and $13 \mathrm{~mm} \mathrm{Hg}$, respectively; fundus examination showed a $\mathrm{C} / \mathrm{D}$ ratio of 0.4 in both eyes (Fig. 2).

\section{Case 3}

63-year-old female patient with a 9-year diagnosis of open-angle glaucoma in treatment with latanoprost and timolol/dorzolamide combination: VA was 20/25 in both eyes; anterior segment evaluation revealed mild nuclear sclerosis; IOP was $14 \mathrm{~mm} \mathrm{Hg}$ in RE and $13 \mathrm{~mm} \mathrm{Hg}$ in LE; fundus examination showed a C/D ratio of 0.3 in the $\mathrm{RE}$ and 0.4 in the LE (Fig. 3).

\section{Case 4}

59-year-old female patient with a 5-year diagnosis of open-angle glaucoma in treatment with latanoprost: VA was 20/25 in both eyes; the slit-lamp examination was normal in both eyes; IOP was $16 \mathrm{~mm} \mathrm{Hg}$ in RE and $18 \mathrm{~mm} \mathrm{Hg}$ in LE; fundus examination showed a cup/disc ratio of 0.5 in both eyes (Fig. 4).

\section{Case 5}

67-year-old female patient with a 12-year diagnosis of open-angle glaucoma in treatment with timolol/dorzolamide combination: VA was $20 / 25$ in both eyes; both eyes presented intraocular lens; IOP was $17 \mathrm{~mm} \mathrm{Hg}$ and $18 \mathrm{~mm} \mathrm{Hg}$, respectively; fundus examination showed a $\mathrm{C} / \mathrm{D}$ ratio of $0.4 \mathrm{in} \mathrm{RE}$ and 0.4 in LE (Fig. 5).

\section{Case 6}

71-year-old male patient: VA was 20/25 in both eyes; anterior segment evaluation revealed intraocu- 
A

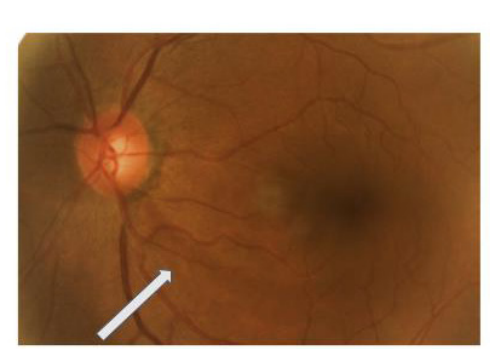

D



B

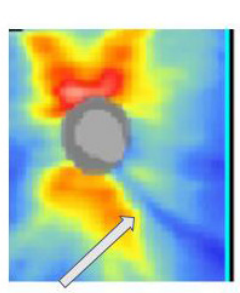

c

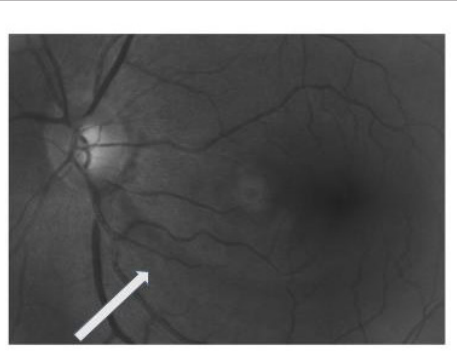

E

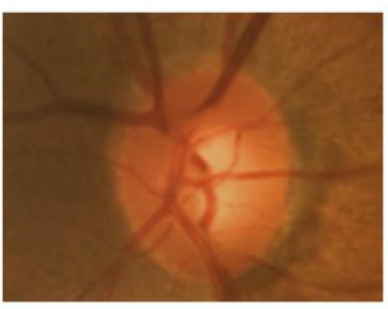

$\mathbf{F}$

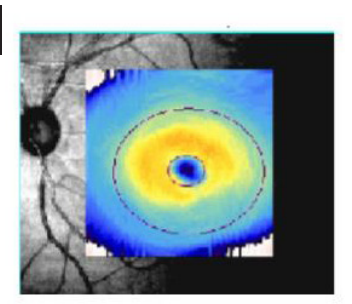

G

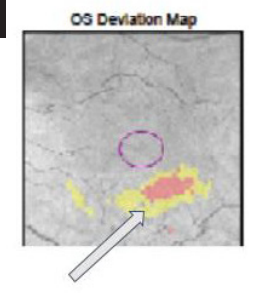

FIGURE 2. A. Left eye (LE) color fundus optic nerve image showing inferior retinal nerve fiber layer (RNFL) defect (white arrow); B. RNFL thickness map showing inferior RNFL defect (white arrow); C. LE red-free optic nerve image showing inferior RNFL defect (white arrow); D. RNFL deviation map showing mild inferior RNFL defect (white arrow); E. First plane physiological cup/disc ratio; F. Ganglion cell complex (GCC) thickness without raphe sign; G. GCC deviation map showing a mild compromise

A



D

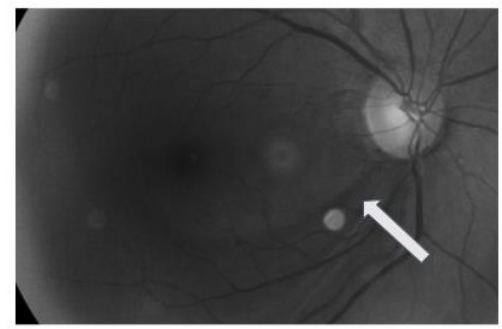

B

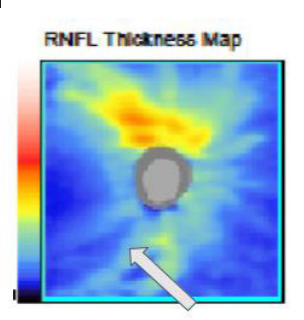

E



C

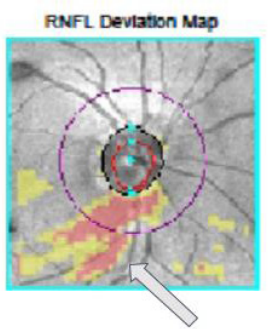

$\mathbf{F}$



G

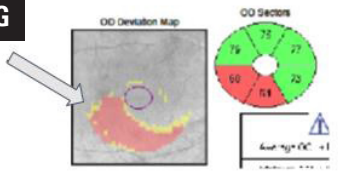

FIGURE 3. A. Right eye (RE) optic nerve color image showing inferior retinal nerve fiber layer (RNFL) defect (white arrow);

B. RNFL thickness map showing inferior RNFL defect (white arrow); C. RNFL deviation map showing inferior RNFL defect (white arrow); D. RE red-free image showing inferior RNFL defect (white arrow); E. First plane, RE physiologic cup/disc ratio; F. Ganglion cell complex (GCC) thickness map showing positive raphe sign (respects horizontal midline) (white arrow); G. GCC deviation map and sectors showing inferior sectors thinning (white arrow)

lar lens in both eyes; IOP was $14 \mathrm{~mm} \mathrm{Hg}$ in RE and $14 \mathrm{~mm} \mathrm{Hg}$ in LE; fundus examination showed a C/D ratio of 0.5 in both eyes (Fig. 6).

\section{Case 7}

49-year-old African descendant male patient with a 2-year history of primary open-angle glaucoma 


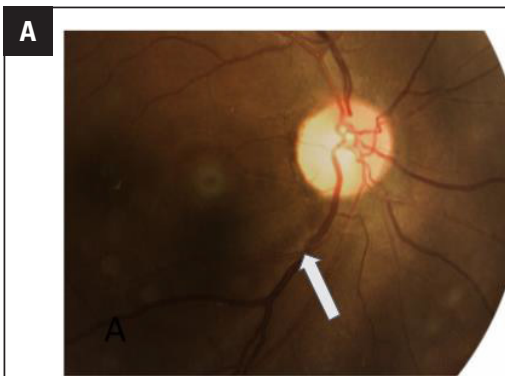

$\mathbf{E}$



B

\section{RNFL Theresses Map}

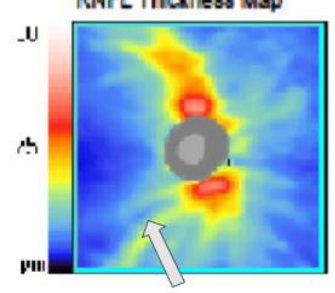

$\mathbf{F}$

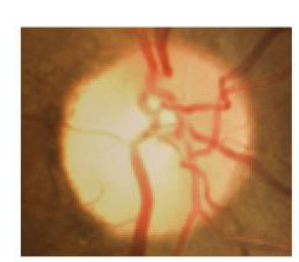

\section{C}

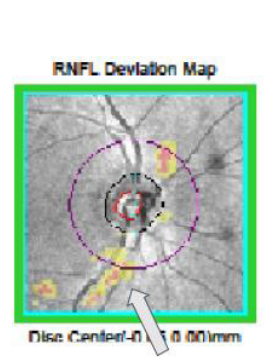

G

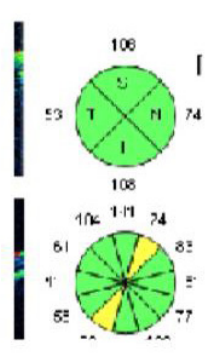

H
D
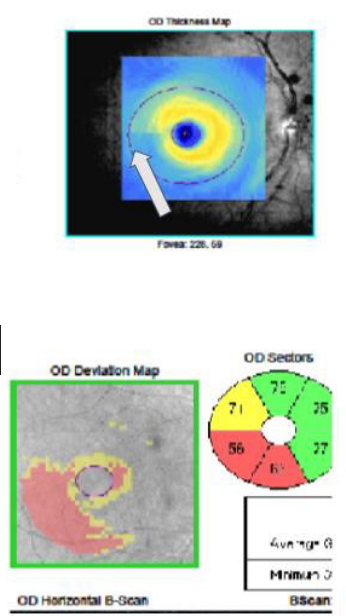

FIGURE 4. A. Right eye (RE) red color image showing inferior retinal nerve fiber layer (RNFL) defect (white arrow); B. RNFL thickness map showing inferior RNFL defect (white line); C. RNFL deviation map showing inferior thinning (white arrow); D. Ganglion cell complex (GCC) thickness map showing positive temporal raphe sign (respects the midline) (white arrow); E. RE red-free optic nerve image showing inferior RNFL defect (white arrow); F. First plane, right optic nerve with a physiological cup/disc ratio; G. RNFL meridians showing inferior thinning (white arrow); H. GCC deviation map and meridians showing inferior thinning (white arrow)

\section{A}

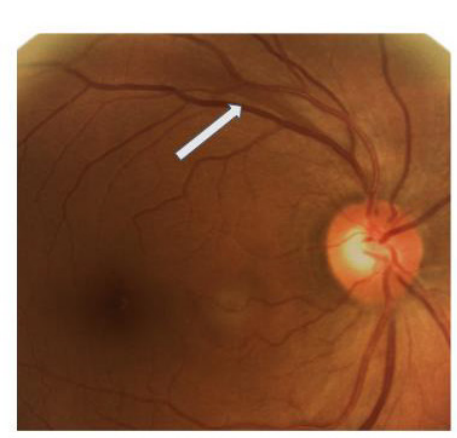

D

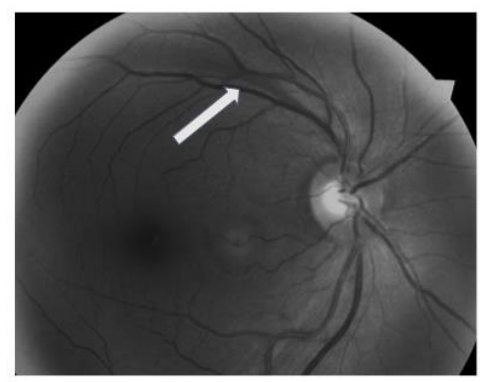

B

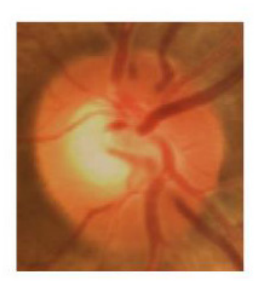

C



E

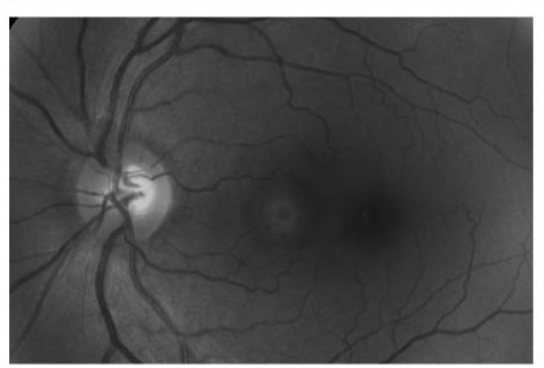

FIGURE 5. A. Right eye (RE) color fundus image showing superior retinal nerve fiber layer (RNFL) defect (white arrow); B. First plane, right optic nerve showing a physiologic cup/disc ratio; C. Left eye (LE) normal color fundus image; D. RE red-free image showing superior RNFL defect (white arrow); E. LE normal red-free image

in treatment with latanoprost and timolol/dorzolamide combination: VA was 20/20 in both eyes; the slit-lamp examination was unremarkable in both eyes; IOP was $12 \mathrm{~mm} \mathrm{Hg}$ in RE and $14 \mathrm{~mm} \mathrm{Hg}$ in $\mathrm{LE}$; fundus examination showed a $\mathrm{C} / \mathrm{D}$ ratio of 0.2 in both eyes (Fig. 7-9). 


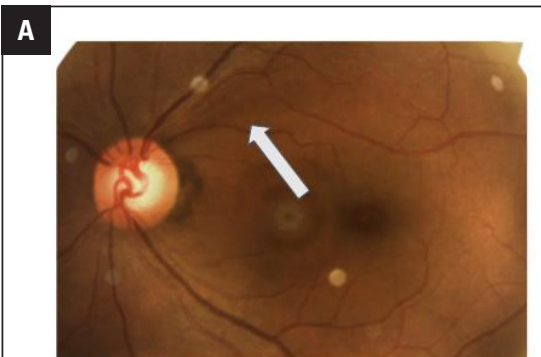

E

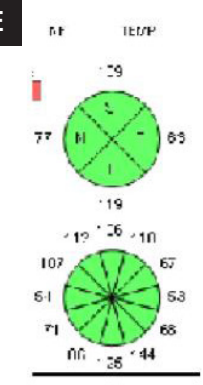

B

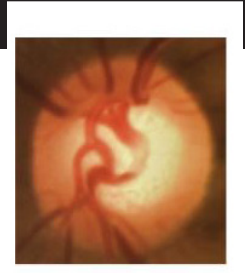

$\mathbf{F}$

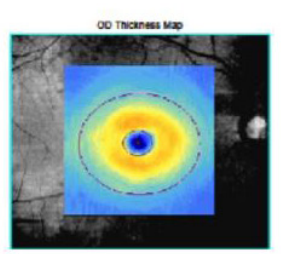

C

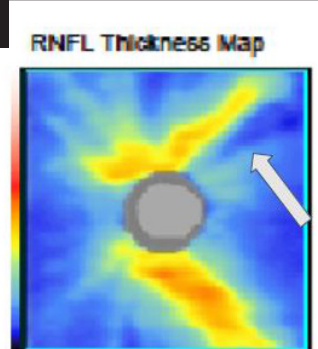

D

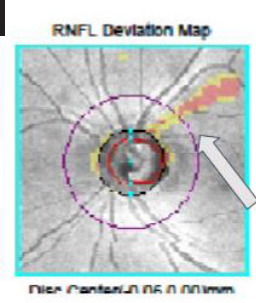

G



H

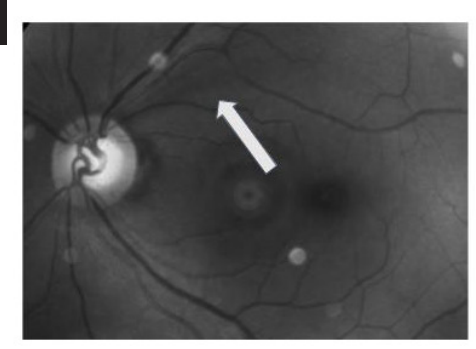

FIGURE 6. A. Left eye (LE) red color image showing superior retinal nerve fiber layer (RNFL) defect (white arrows); B. First plane, left optic nerve with a physiological cup/disc ratio; C. RNFL thickness map showing superior RNFL defect (white arrows); D. RNFL deviation map showing superior thinning (white arrow); E. Normal RNFL meridians; F. Ganglion cell complex (GCC) normal thickness map;

G. GCC normal deviation map and meridians map; H. LE red-free optic nerve image showing superior RNFL defect (white arrow)

A

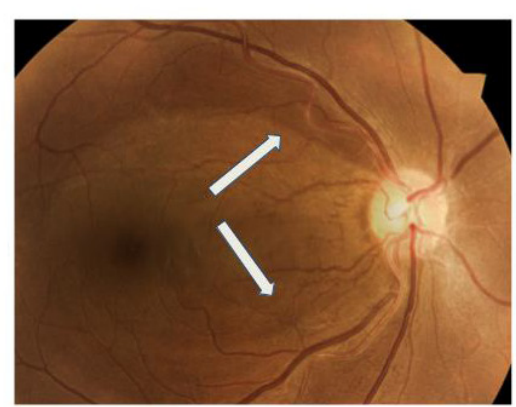

D

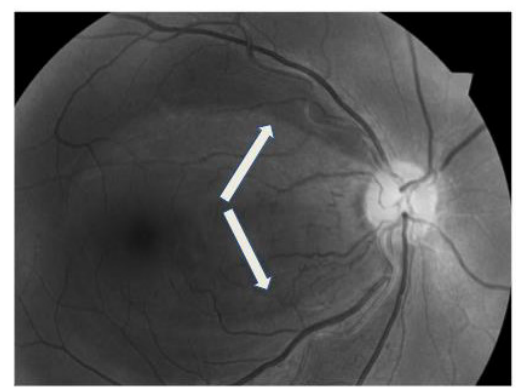

B

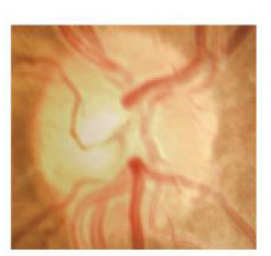

E

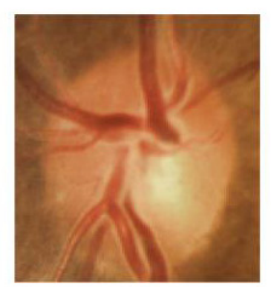

c



$\mathbf{F}$

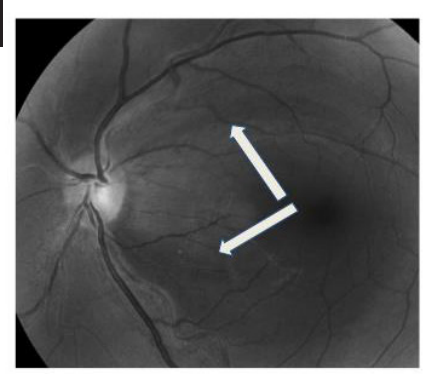

FIGURE 7. A. Right eye (RE) color image showing superior and inferior retinal nerve fiber layer (RNFL) defect (white arrows); B. RE color fundus image showing physiologic cup/disc ratio; C. Left eye (LE) color image showing superior and inferior RNFL (white arrows);

D. RE red-free image showing superior RNFL defect (white arrows); E. LE normal color fundus image showing a physiologic cup/disc ratio; F. LE red-free image showing superior and inferior RNFL defect (white arrows)

\section{DISCUSSION}

Retinal nerve fiber layer defects were first described in 1913 and later in 1917 by Vogt [5]. He described the enhanced visibility of striated retinal nerve-fiber reflexes with an ophthalmoscopic illuminating system from which red (long) wavelengths of 
A

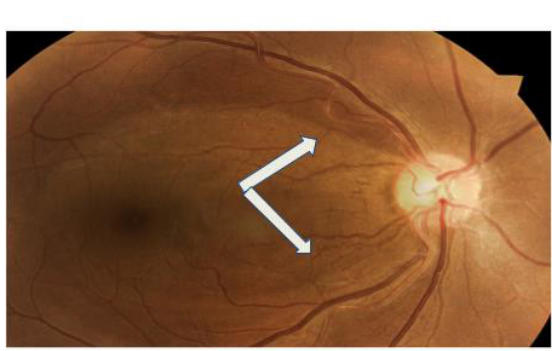

B

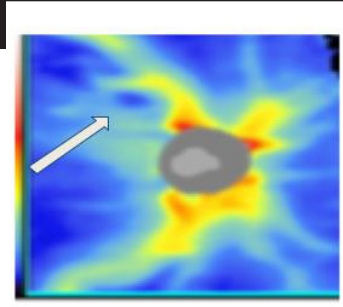

C

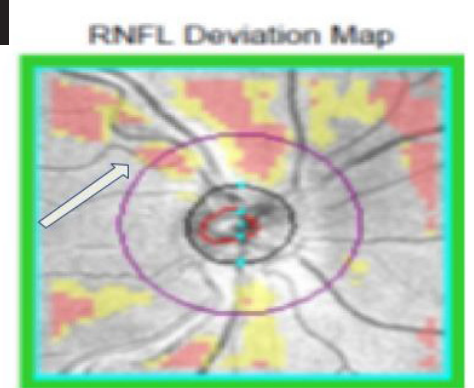

D

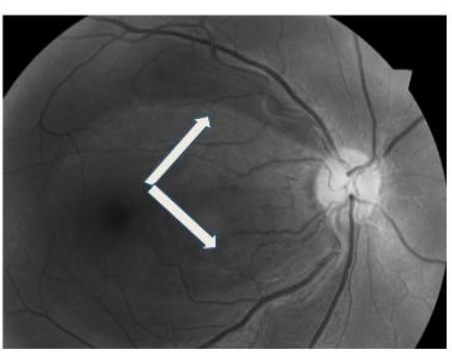

E

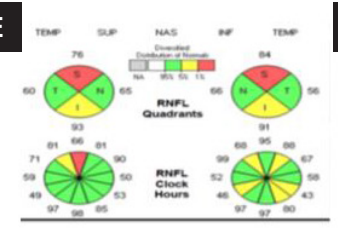

$\mathbf{F}$



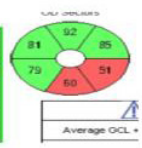

G

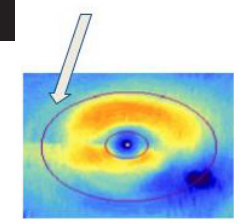

FIGURE 8. A. Right eye (RE) red color image showing superior and inferior retinal nerve fiber layer (RNFL) defect (white arrow);

B. RNFL thickness map showing superior diffuse RNFL defect (white arrow); C. RNFL deviation map showing superior thinning (white arrow); D. RE red-free optic nerve image showing superior and inferior RNFL defect (white arrow); E. RNFL meridians showing superior thinning; F. Ganglion cell complex (GCC) deviation map and meridians showing inferior thinning; G. GCC thickness map showing temporal raphe positive sign (white arrow)

A

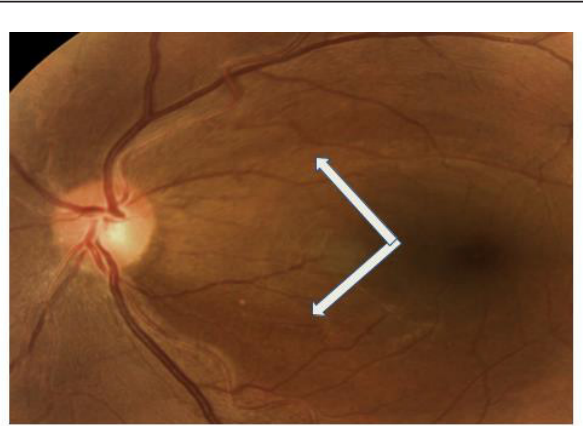

D

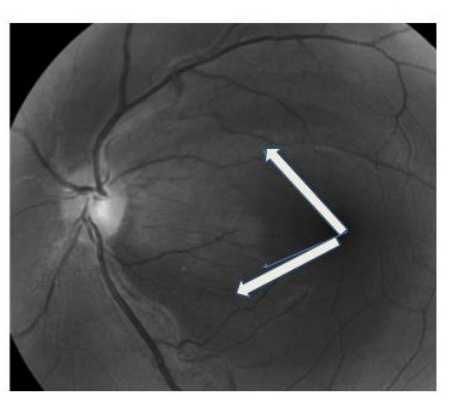

B

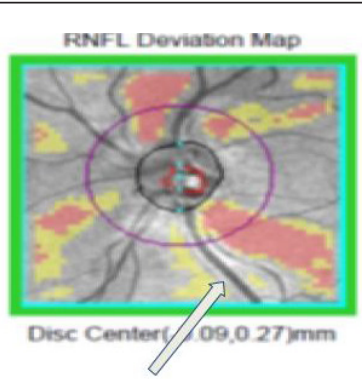

E

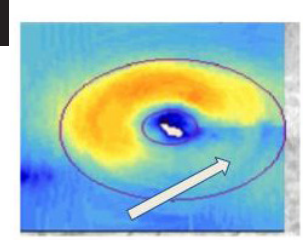

$\mathbf{F}$

C

RNFL. Thickness Map
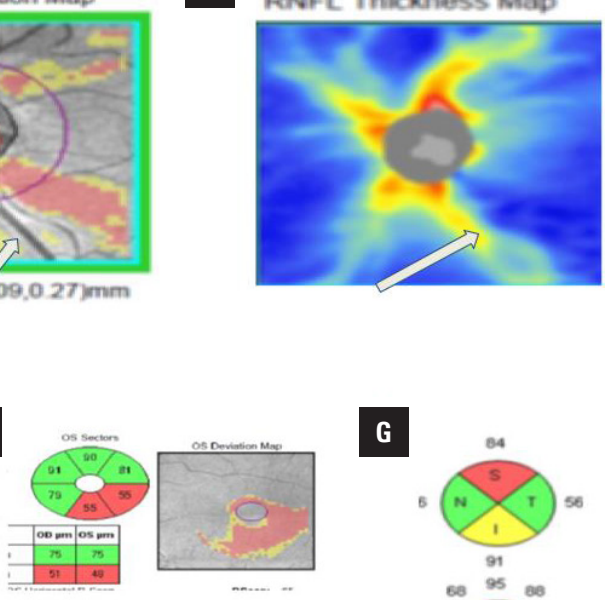

G

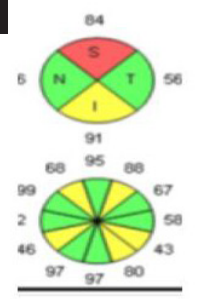

FIGURE 9. A. Left eye (LE) red color image showing superior and inferior retinal nerve fiber layer (RNFL) defect (white arrow);

B. RNFL deviation map showing superior and inferior thinning (white arrow); C. RNFL thickness map showing inferior thinning (white arrow); D. Left eye (LE) red-free optic nerve image showing superior and inferior RNFL defect (white arrow); $\mathbf{E}$. Ganglion cell complex (GCC) thickness map showing temporal raphe positive sign (white arrow); F. GCC deviation map and meridians showing inferior thinning; G. RNFL meridians showing superior thinning 
light were excluded [6]. In healthy eyes, the RNFL has a sparkly, homogeneous, striated appearance and is generally identifiable close to the superior and inferior poles of the optic disc where the RNFL is thickest [7]. The Retinal nerve fiber layer striations represent bands of retinal ganglion cells (RGC) axons separated by Muller cell processes. The highly reflective RGC axon bundles generate a bright component of the striation, and the thick non-reflective septa dividing Muller cell glial produces the dark bands between [8].

The reflectivity of RGC axon bundles is diffuse or localized. The reduction in reflectivity is proportional to a loss of RGC axons [5, 9]. Diffuse axonal loss reduces RNFL striations and increases visibility of the retinal blood vessels, which are generally embedded in the RNFL $[8,9]$. Localized loss is more easily visible because it has sharply demarcated borders $[7,10]$. Localized RNFL defects typically have a wedge-shaped pattern and become narrower as the RNFL bundles converge toward the disc margin [11]. Despite nonglaucomatous optic neuropathies, papilledema optic disc drusen, or ischemic retinopathies occasionally can present with localized RNFL defects, they have high specificity for glaucoma and are not found in healthy eyes.

Retinal nerve fiber layer defects are generally present with glaucoma's other optic nerve characteristics, such as superior or inferior rim thinning, enlarged $\mathrm{C} / \mathrm{D}$ ratio, and B-zone of parapapillary atrophy. However, RNFL defects can also be present in a normal C/D ratio setting. When the optic nerve cup/disc ratio is typical, it is easy to miss an RNFL defect because the observer may assume that the extra disc features are also regular.

It is essential to always evaluate in the slit-lamp, with good mydriasis, the optic nerve, and retina with a green light to detect subtle RNFL defects. High-quality optic nerve color and red-free photographs can help us detect early RNFL defects and avoid misdiagnosis of glaucoma or other optic nerve abnormalities. Optical coherence tomography (OCT) has an excellent diagnostic capability in detecting early phases of glaucoma. Hwang et al. compared the ability of thickness, clock-hour, deviation maps obtained with OCT (Cirrus HD-OCT), and concluded that the RNFL thickness map showed the best performance in detecting photographic RNFL defects. Retinal nerve fiber layer defects can be detected efficiently using the RNFL thickness map [12].

\section{CONCLUSIONS}

Retinal nerve fiber layer defects are one of the earliest signs of glaucoma. These defects are generally present with superior or inferior rim thinning, but they can also be present in eyes with a normal $\mathrm{C} / \mathrm{D}$ ratio. Optical coherence tomography plays an essential role in the early detection of glaucoma in these patients, especially the RNFL, GCC thickness, and deviation map. Early detection of RNFL defects can dramatically change the natural history of a patient with glaucoma.

\section{REFERENCES}

1. Kwon YH, et al. Primary Open-Angle Glaucoma. N Engl J Med. 2009; 360(11): 1113-1124, doi: 10.1056/NEJMra0804630, indexed in Pubmed: 19279343.

2. Hood DC, Kardon RH. A framework for comparing structural and functional measures of glaucomatous damage. Prog Retin Eye Res. 2007; 26(6): 688-710, doi: 10.1016/.jpreteyeres.2007.08.001, indexed in Pubmed: 17889587.

3. Inzelberg R, Ramirez JA, Nisipeanu $P$, et al. Retinal nerve fiber layer thinning in Parkinson disease. Vision Res. 2004; 44(24): 2793-2797, doi: 10.1016/. visres.2004.06.009, indexed in Pubmed: 15342223.

4. Paquet $\mathrm{C}$, Boissonnot M, Roger $\mathrm{F}$, et al. Abnormal retinal thickness in patients with mild cognitive impairment and Alzheimer's disease. Neurosci Lett. 2007; 420(2): 97-99, doi: 10.1016/j.neulet.2007.02.090, indexed in Pubmed: 17543991.

5. Hoyt WF, Schlicke B, Eckelhoff RJ. Fundoscopic appearance of a nerve-fibre-bundle defect. Br J Ophthalmol. 1972; 56(8): 577-583, doi: 10.1136/bjo.56.8.577, indexed in Pubmed: 5079403.

6. Quigley HA. Examination of the retinal nerve fiber layer in the recognition of early glaucoma damage. Trans Am Ophthalmol Soc. 1986; 84: 920-966, indexed in Pubmed: 3109098.

7. Radius RL, Anderson DR. The histology of retinal nerve fiber layer bundles and bundle defects. Arch Ophthalmol. 1979; 97(5): 948-950, doi: 10.1001/archopht.1979.01020010506027, indexed in Pubmed: 109068.

8. Airaksinen $P$, Drance $S$, Douglas $G$, et al. Diffuse and Localized Nerve Fiber Loss in Glaucoma. Am J Ophthalmol. 1984; 98(5): 566-571, doi: 10.1016/0002-9394(84)90242-3, indexed in Pubmed: 6496612.

9. Hoyt WF, Schlicke B, Eckelhoff RJ. Fundoscopic appearance of a nerve-fibre-bundle defect. Br J Ophthalmol. 1972; 56(8): 577-583, doi: 10.1136/bjo.56.8.577, indexed in Pubmed: 5079403.

10. Tuulonen A, Lehtola J, Airaksinen P. Nerve Fiber Layer Defects with Normal Visual Fields. Ophthalmology. 1993; 100(5): 587-598, doi: 10.1016/s0161-6420(93)31598-8, indexed in Pubmed: 8493001.

11. Jonas JB, Schiro D. Localized retinal nerve fiber layer defects in nonglaucomatous optic nerve atrophy. Graefes Arch Clin Exp Ophthalmol. 1994; 232(12): 759-760, doi: 10.1007/BF00184280, indexed in Pubmed: 7890191.

12. Hwang YH, Kim YY, Kim HKi, et al. Ability of cirrus high-definition spectral-domain optical coherence tomography clock-hour, deviation, and thickness maps in detecting photographic retinal nerve fiber layer abnormalities. Ophthalmology. 2013; 120(7): 1380-1387, doi: 10.1016/j.ophtha.2012.12.048, indexed in Pubmed: 23541761.

13. Foster PJ, Buhrmann R, Quigley HA, et al. The definition and classification of glaucoma in prevalence surveys. Br J Ophthalmol. 2002; 86(2): 238-242, doi: 10.1136/bjo.86.2.238, indexed in Pubmed: 11815354. 